\title{
Psychodynamische Psychotherapie mit Psychoseerkrankten
}

\author{
Jürgen Körner
}

Online publiziert: 17. November 2015

(C) Springer-Verlag Berlin Heidelberg 2015

Vier Jahre nach dem Themenheft „Psychoanalytische Konzepte der Psychosentherapie“ legt Forum der Psychoanalyse erneut eine Sammlung von Beiträgen vor, die sich der psychoanalytisch begründeten Arbeit mit psychotisch erkrankten Menschen widmen. Das Anliegen der Autoren von damals war es, psychodynamische Auffassungen zur Ätiologie und zu den therapeutischen Behandlungsmöglichkeiten psychotisch Erkrankter zusammenzutragen und den damals dominierenden somatischen Erklärungen und medikamentösen Therapiemethoden gegenüberzustellen. Ihre Arbeiten trugen wesentlich dazu bei, dass sich die Methoden psychodynamischer Psychotherapie mit psychotisch erkrankten Menschen heute nicht mehr legitimieren müssen; auch die unfruchtbaren Alternativen zu den medikamentösen Therapien einerseits und den verhaltenstherapeutischen Methoden andererseits scheinen weitgehend überwunden zu sein.

Die Beiträge des vorliegenden Themenhefts bringen die Diskussion über die Ätiologie und die Therapie psychotisch Erkrankter auf einen neuen Stand. Günter Lempa unternimmt den Versuch, weit auseinanderliegende Theorien von Freud, Lacan, Bion und Winnicott heranzuziehen, um die für die Schizophrenie charakteristische Störung in der Konstitution und der Verarbeitung der Realität zu erklären. Im Einklang mit dem Dilemma-Konzept von Stavros Mentzos beschreibt er, wie der psychotisch Erkrankte in der Auseinandersetzung mit einer schwer erträglichen Realität scheitert und doch versucht, sein Ich mit Symptomen des Wahns und der Halluzinationen zu reorganisieren.

Christiane Montag referiert aktuelle Konzepte der Mentalisierung und untersucht, wie die psychopathologischen Kernsymptome Ausdruck von schwerwiegenden Mentalisierungsstörungen sein können, beginnend bei basalen Störungen der symbolischen und sprachlichen Repräsentation, des Wahrnehmens und Erkennens von

Prof. Dr. disc. pol. J. Körner $(\bowtie)$

Cimbernstr. 28,

14129 Berlin, Deutschland

E-Mail: juergen.koerner@ipu-berlin.de 
Affekten bis hin zu dem Unvermögen, eigene Erfahrungen zu kohärenten biografischen Erzählungen zu integrieren. Die Autorin zeigt anhand von Fallbeispielen, wie die mentalisierungsbasierte psychotherapeutische Arbeit mit psychotisch Erkrankten gelingen kann.

Dorothea von Haebler greift die zuvor dargestellten Konzepte von Mentzos, Lempa und Montag auf und entwickelt eine spezielle, für die psychotherapeutische Behandlung psychotisch erkrankter Menschen geeignete Methode. Ihr Entwurf berücksichtigt die unterschiedlichen Zustände der Ich-Organisation dieser Patienten, auf die sich der psychodynamische Psychotherapeut einstellen muss. Da diese Zustände auch kurzfristig wechseln können, bedarf es einer flexiblen therapeutischen Strategie, weswegen die Autorin ihr Behandlungsmanual als eine Sammlung unterschiedlicher „Werkzeuge“ versteht, die man wie einen „Werkzeugkasten“ zusammensetzen kann. Grundlage einer gelingenden Psychosentherapie ist allerdings eine spezifische Haltung des Therapeuten: Einerseits muss er offen sein in dem Sinne, dass er darauf verzichten kann, bestimmte Ziele mit seinem Patienten zu verfolgen, stattdessen aber damit rechnen muss, ganz unerwarteten Ideen und Entwicklungen zu begegnen. Andererseits soll er „Festigkeit“ zeigen und einen geschützten Raum anbieten, sodass sich der Patient, der oft genug unter einem sehr starken Druck steht, sicher fühlen kann.

Stavros Mentzos erläutert seine empirisch gestützte, psychodynamische Hypothese, der zufolge die psychotischen Symptome nicht allein als Störung, sondern auch als aktive Versuche verstanden werden können, mit denen das Subjekt einen drohenden Verlust des Ich abzuwenden und zu bewältigen versucht. Diese Patienten standen in ihrer Kindheit oft vor der unlösbaren Aufgabe, sich zwischen symbiotischer Gebundenheit an einen Elternteil einerseits und dem Wunsch nach Befreiung des Selbst andererseits entscheiden zu müssen. In diesem kaum erträglichen Dilemma greifen sie zu psychotischen Symptomen, mit denen sie die Gefahr des drohenden Ich-Verlustes abwehren. Diese Hypothese ist insofern für die Methode psychodynamischer Arbeit mit psychotisch Kranken so fruchtbar, weil sie einen therapeutischen Zugang innerhalb der Patient-Therapeut-Beziehung eröffnet.

Der Beitrag von Stavros Mentzos steht an erster Stelle der nun folgenden vier Texte zur psychodynamischen Psychosentherapie. Ihm kommt eine Schlüsselstellung zu, weil die dort vorgestellten Hypothesen den anderen Autoren bekannt waren, sodass sie sich in ihren Texten auf sie beziehen konnten. Leider hat Stavros Mentzos seine Arbeiten an diesem Text nicht abschließen können; er verstarb im Mai 2015. Wir sind dankbar, dass uns das noch unvollendete Manuskript zur Verfügung stand. Dorothea von Haebler und Günter Lempa haben es im Sinne des Autors zu Ende geführt, sodass wir seine Auffassungen in der aktuellsten Version drucken konnten. 